\title{
A QOS Based Routing in Mobile Ad-Hoc Networks
}

\author{
Ronal Benitto Dhas .A ${ }^{1}$, Ruby. ${ }^{2}$ \\ PG Scholar, Dept of Computer Science, DMI College of Engineering ${ }^{1}$ \\ Assistant professor, Dept of Computer Science, DMI College of Engineering ${ }^{2}$
}

\begin{abstract}
With the increasing usage of mobile devices, Mobile ad-hoc network (MANET) has been proven to be an essential part of wireless communication and there are many opportunities for using MANET in real time application. Quality of service is an indispensable for implementing this real time application. Since MANET is infrastructure less network and nodes are moving dynamically, ensuring of QoS parameter like delay, throughput, etc are challenging. In this paper, QoS based routing protocol is proposed with the aim of increasing QoS support in MANET communication. It incorporates following: 1) a Game theory based neighbour selection algorithm to meet the transmission requirement. 2) A packet scheduling algorithm to reduce the transmission delay. 3) A segment resizing algorithm that adjusts the segment size based on the node's mobility. Analytical results show that the protocol provides good performance in terms of delay and throughput.
\end{abstract}

Keywords: Mobile adhoc networks, Routing algorithms, Quality of service, Game theory

\section{INTRODUCTION}

Mobile Ad-Hoc network is a collection of wireless mobile nodes that self-organizes itself in dynamic packet in its packets stream based on the neighbour's and temporary network topologies. Nodes can connect and mobility.

leave the network at anytime and should be in position to relay traffic. Each node in the network has the ability to independently adapting its operation based on the current sl€Si with the objective of maximizing its utility ui. The environment according to predetermined algorithms and utility function ui(s): $S \rightarrow R$ represents each player's protocols. Due to the dynamic nature of Mobile Ad-Hoc sensitivity to everyone's actions.

Networks, there are many issues which need to be tackled According to the above, a game can be modelled as $\mathrm{G}=$ and one of the areas for improvement is Quality of Service (QoS) routing. When we consider QoS routing, the routing protocols have to ensure that the QoS requirements are met. QoS routing protocols have been proposed for MANETs that create routes formed by nodes and links that reserve their resources to fulfil QoS requirements. Here how to guarantee QoS based routing can be transformed to the problem of how to schedule the neighbour resources between nodes to ensure QoS of packet routing and some problem of how to select intermediate nodes that can guarantee the QoS of the packet transmission and how a source node assigns traffic to the intermediate nodes to ensure their scheduling feasibility. Few challenges faced in providing QoS are persistently changing environment, unrestricted mobility which causes recurrent path breaks.

QoS based distributed routing protocol is developed for MANET with the aim of reducing the delay and increasing the throughput while transmitting the data, which has following contribution

Game theory based neighbour selection algorithm: This algorithm selects qualified neighbour from the set of all available neighbours

Distributed scheduling algorithm: After qualified neighbours are identified, based on enhanced edf algorithm packets routes are scheduled.
$\{\mathrm{P}, \mathrm{A}, \mathrm{S}\}$

Where: $\mathrm{P}$ denotes set of players.

A denotes available resource

$\mathrm{S}$ denotes the strategies for players.

Game theory can be applied to the modelling of an ad hoc network at the physical layer (distributed power control control) and network layer (packet forwarding).

\section{NETWORK MODEL:}

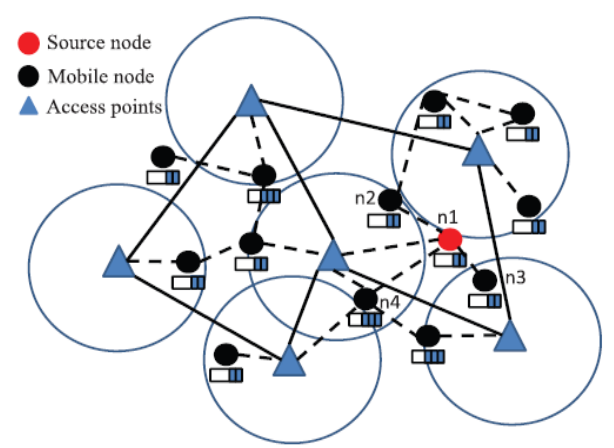

Figure 1: network model of MANET and waveform adaptation), link layer (medium access 
We consider a mobile ad-hoc network with number of base station spreading over the network. Here the mobile nodes $(\mathrm{N})$ moving around the network, each node is considered as $\operatorname{ni}(1<\mathrm{i}<\mathrm{N})$ and base stations are considered as a access points. When a source node $\mathrm{n} 1$ wants to upload files to an Internet server throughout the APs, it can choose to send packets to the APs directly by itself or require its neighbour nodes $\mathrm{n} 2, \mathrm{n} 3$ or $\mathrm{n} 4$ to assist the packet transmission. We assume that queuing occurs only at the output ports of the mobile nodes. After a mobile node generates the packets, it tries to transmit the packets to its nearby APs that can guarantee the QoS requirements. If it fails (e.g., out of the transmission range of APs or in a hot/dead spot), it relies on its neighbours that can guarantee the QoS requirements for relaying packets to APs. Relaying for a packet stream can be modelled as a process, in which packets from a source traverse a number of queuing servers to some APs. In this model, the problem of how to guarantee QoS routing can be transformed to the problem of how to schedule the neighbour resources between nodes to ensure QoS of packet routing. Scheduling feasibility is the ability of a node to guarantee a packet to arrive at its destination within QoS requirements

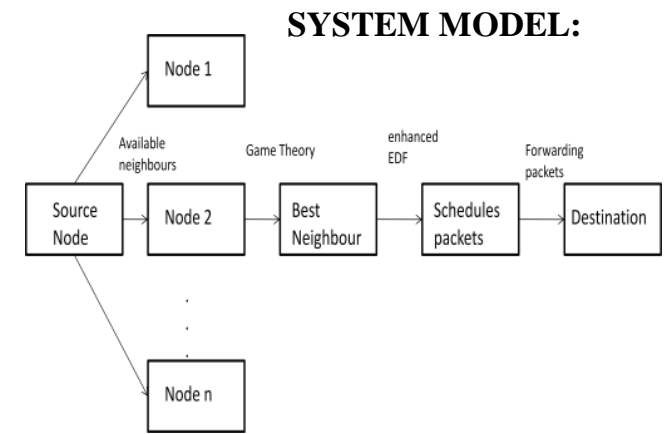

Figure 2: architecture diagram for packet transmission Game theory based neighbour selection algorithm:

Since short delay and throughput are the major real-time QoS requirement for traffic transmission, Qos based protocol incorporates game theory algorithm for neighbour selection. In this algorithm, the source node broadcast the message to all available nodes with its bandwidth. The neighbouring node which receives the message replies to the source node. The reply message has information about available resources for checking packet scheduling feasibility, available bandwidth, and energy. The source node identifies the selfish node by checking the transmission history of all replied node, and choose the best neighbour from them. Selfishness is generally detrimental to overall network performance. Selfish node refusing to forward packets for its neighbours which increases the QoS delay and throughput. In Game theory source node is rejecting this selfish node by checking its transmission history. Here $\mathrm{Sp}(\mathrm{i})$ to denote the size of the packet steam from node ni, use Wi to represents the bandwidth of node $\mathrm{i}$, and Ta(i) to denote the packet arrival interval from node ni then the bandwidth of the packet calculated by

$$
\mathrm{Wi}=\mathrm{Sp} / \mathrm{Ta}
$$

The QoS of the packets going through node $\mathrm{n}$ can be satisfied if

$$
\mathrm{Sp}(1) / \mathrm{Ta}(1)+\mathrm{Sp}(\mathrm{j}) / \mathrm{Ta}(\mathrm{j})+\ldots \ldots . . \mathrm{Sp}(\mathrm{m}) / \mathrm{Ta}(\mathrm{m})=\mathrm{Wi}
$$

\section{Distributed scheduling:}

Neighbour selection solves the problem of how to select an intermediate node that can guarantee the Qos of the packet communication. To further reduce the packet transmission delay, enhanced edf based packet scheduling is proposed in packet transmission. Edf assigns earlier generated packets to forwarders with higher priority while assigns more recently generated packets to forwarders with lower priority so that the transmission delay of an entire packet stream can be reduced.

We use $t$ to denote the time when a packet is generated, and use $\mathrm{T}_{\mathrm{QoS}}$ to denote the delay QoS requirement. Let $\mathrm{W}_{S}$ and $\mathrm{W}_{\mathrm{I}}$ denote the bandwidth of a source node and an intermediate node respectively, we use $\mathrm{T}_{\mathrm{S} \rightarrow \mathrm{I}}=\mathrm{Sp} / \mathrm{W}_{\mathrm{S}}$ to denote the transmission delay between a source node and an intermediate node, and $\mathrm{T}_{\mathrm{I}_{\rightarrow} \mathrm{D}}=\mathrm{Sp} / \mathrm{W}_{\mathrm{I}}$ to denote the transmission delay between an intermediate node and destination. Let $\mathrm{Tw}$ denote the packet queuing time, $\mathrm{T}_{\mathrm{Q}}$ denote the pending queue. This pending queue maintains the packets which are generated but not scheduled by the edf order. The queuing delay requirement is calculated as $\mathrm{Tw}<\mathrm{T}_{\mathrm{QoS}} \mathrm{T}_{\mathrm{S}_{\rightarrow} \mathrm{I}} \mathrm{T}_{\mathrm{I}_{\rightarrow} \mathrm{D}}$. The source node needs to calculate $\mathrm{Tw}$ of each intermediate node to select intermediate nodes that can send its packets by the deadline, i.e., that can satisfy $\mathrm{Tw}<\mathrm{T}_{\mathrm{QoS}} \mathrm{T}_{\mathrm{S}_{\rightarrow} \mathrm{I}} \mathrm{T}_{\mathrm{I}_{\rightarrow} \mathrm{D} \text {. The }}$ queuing time $\mathrm{Tw}$ of a packet with priority $\mathrm{x}$ is estimated by

$$
\mathrm{Tw}=€\left(\mathrm{~T}_{\mathrm{I}_{\rightarrow} \mathrm{D}} \cdot\left[\mathrm{T}_{\mathrm{Q}} / \mathrm{T}_{\mathrm{a}}\right]\right)
$$

$\mathrm{T}_{\mathrm{I}_{\rightarrow} \mathrm{D}}$ and Ta respectively denote the transmission delay and arrival interval of a packet with the $\mathrm{x}^{\text {th }}$ priority. $\left[\mathrm{T}_{\mathrm{Q}} / \mathrm{T}_{\mathrm{a}}\right]$ is the number of packets arriving during the packet's queuing time Tw, which are sent out from the queue before this packet. After calculates the Tw of each intermediate node, the source node chooses the intermediate node that should satisfies

$$
\mathrm{Tw}<\mathrm{T}_{\mathrm{Qos}} \mathrm{T}_{\mathrm{S}_{\rightarrow} \mathrm{D}} \mathrm{T}_{\mathrm{I}_{\rightarrow} \mathrm{D}}
$$

Consider an example a source node generates three packets $\mathrm{p} 0, \mathrm{p} 1$, and $\mathrm{p} 2$ with the same size at times $\mathrm{t} 0$, $\mathrm{t} 1$, and $\mathrm{t} 2$ here $(\mathrm{t} 0<\mathrm{t} 1<\mathrm{t} 2)$, respectively. A packet $\mathrm{p}$ 's total transmission delay equals: $T_{S_{\rightarrow} I}$ (i) $+\mathrm{Tw}$ (i) $+T_{I_{\rightarrow} D}$ (i). Since all these packets are created by the same node, the delay from the source node to each intermediate node $\mathrm{T}_{\mathrm{S}_{\rightarrow} \mathrm{I}}(0)$, $\mathrm{T}_{\mathrm{S}_{\rightarrow} \mathrm{I}}(1)$, and $\mathrm{T}_{\mathrm{S}_{\rightarrow} \mathrm{I}}(2)$ are almost the same. To simplify the analysis, we suppose $\mathrm{T}_{\mathrm{I}_{\rightarrow} \mathrm{D}}(0)=\mathrm{T}_{\mathrm{I}_{\rightarrow} \mathrm{D}}(1)=\mathrm{T}_{\mathrm{I}_{\rightarrow} \mathrm{D}}(2)$. If the queuing delay in each intermediate node satisfies $\mathrm{Tw}$ (0) $>\mathrm{Tw}(1)>\mathrm{Tw}$ (2), then packet p0 should be sent to the first intermediate node, packet p1should be sent to the second intermediate node, and packet $\mathrm{p} 2$ should be sent to the next intermediate node. As an end result, the final packet delivery time for the three packets from the intermediate nodes to the destination node can be reduced. 
Segment Resizing:

In a mobile ad hoc network, the transmission link between two nodes is broken frequently. This makes more delay to retransmit the packets and degrades the QoS of the transmission. On the other hand, a node in a highly
dynamic network has higher probability to meet different mobile nodes, which is beneficial to resource scheduling. The space utility of an intermediate node that is used for transmission. Since Two-hop and QOD only have forwarding a packet $\mathrm{p}$ is $\mathrm{Sp}=\mathrm{Wi} / \mathrm{Ta}$. That is, reducing hops in the routing paths to APs, the shortest path have packet size can increase the scheduling feasibility of an lower probability to break down. Even if a link breaks, the intermediate node and reduces packet dropping possibility. source node can quickly choose another forwarder. However, we cannot make the size of the packet too small Therefore, node mobility does not really have an effect on because it makes more packets to be transmitted and these two protocols. E-AODV has much smaller QoS higher packet overhead. Based on this rationale, we throughput than QOD with different node mobility. This is propose a mobility-based packet resizing algorithm in this because in E-AODV, the routing resources in every link section. The fundamental idea is that the larger size are reserved for QoS traffic. In a highly dynamic mobile packets are assigned to lower mobility intermediate nodes network, the reserved links are constantly broken down, and smaller size packets are assigned to higher mobility which makes invalid reservation problem and forcing the intermediate nodes, which raises the QoS-guaranteed source node to search for a new path to an access point. packet transmissions. Specifically, as the mobility of a The delay resulted from the path searching degrades the node increases, the size of a packet $\mathrm{Sp}$ sent by a node to its ability to meet the QoS requirements. The race condition neighbour nodes i decrease as following:

$\operatorname{Sp}($ new packet $)=(\alpha /$ ei $) \operatorname{Sp}($ original packet $)$

Here $\mathrm{Sp}$ is the size of the packet and $\alpha$ is the scaling parameter.

\section{Performance results}

This section demonstrates the distinguishing properties of QOS based routing compared to E-AODV, SMultihop, Two-hop, through simulations on NS-2. EAODV is a resource reservation-based routing protocol for QoS routing in MANETs. This protocol expands AODV by adding information of the maximum delay and minimum available bandwidth of each neighbour in a node's routing table. To apply E-AODV in mobile networks, we let a source node search for the QoS guaranteed path to an access point. The Intermediate nodes along the path reserve the resources for the source node. In S-Multihop, a node always transmits a packet to a next hop node that has small buffer usage than itself until the packet reaches an AP. In Two-hop, the source node chooses direct transmission and to forward packets to APs. In the simulation, Six APs with IEEE 802.11 MAC protocol are consistently distributed in the area. We randomly chose two source nodes to send packets to APs in every $10 \mathrm{~s}$. A node's traffic is created with constant bit rate (CBR). The generation rate of the CBR traffic is $100 \mathrm{~kb} / \mathrm{s}$. unless otherwise mentioned; the speeds of the nodes were randomly Selected from $[1-40] \mathrm{m} / \mathrm{s}$

\section{Performance with Various Mobility Speeds}

In this experiment, a node's mobility speed was arbitrarily selected from $[1, \mathrm{x}] \mathrm{m} / \mathrm{s} \mathrm{x}=[1 ; 4 ; 6 ; 8 ; 10]$. Figure plots the QoS throughput of all systems versus the node mobility. It shows that the QoS throughputs increase ratio of all systems decrease as node mobility increases. This is because increasing mobility causes higher frequent link breakages, which makes to more packet drops. problem further decreases the QoS throughput as the same resources are reserved for different source nodes at the same time. Then some nodes cannot obtain the resources as scheduled. As the result, QoS of the packet traffic in EAODV is very difficult to guarantee in a highly dynamic network. Since a node in S-Multihop forwards a packet to the next hop with smaller buffer usage without reserving resource, meanwhile, in S-Multihop, as several source nodes may send packets to the node with smaller buffer usage at the same time, the node is very easily crammed. . Although the routing path length in two-hop is always two as QOD, as Two-hop only concerns bandwidth of node in packet forwarding rather than buffer usage, it may suffer buffer congestion in the selected node with high bandwidth.

Therefore, S-Multihop generates higher QoS throughput than two-hop in a low-mobility network. However, S-Multihop suffers strictly from node mobility due to it long paths while Two-hop is mobility resilient due to its short path. Therefore, S-Multihop produces less QoS throughput than two-hop in high node mobility.

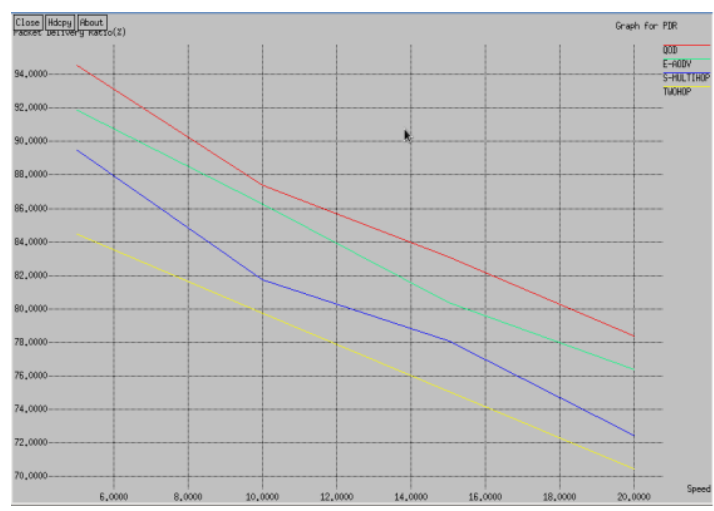

Figure3: packet throughput ratio versus different speed 


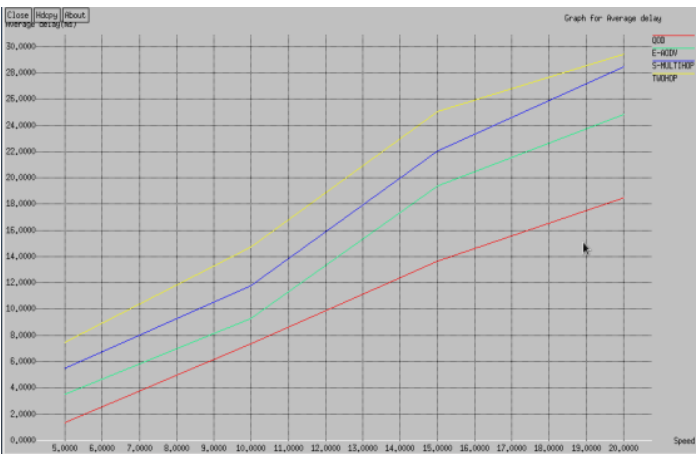

\section{Figure4: average delay versus different speed}

In QoS based distributed routing the intermediate nodes regularly reports their queuing status to the source node. The source node schedules the packets to the neighbour nodes based on their current space utilities. Therefore, there is no need for retransmission caused by invalid reservation. Moreover, since every middle node can receive scheduled packets for the forwarding transmission, and the same resource will not be assigned to more than one source node at the same time, the race condition problem can be avoided. Although both QOD and Two-hop have at most two hops from source nodes to APs, QOD constantly creates higher QoS throughput than two-hop. This is because of two reasons. First, QOD vigorously schedules the packets to the neighbours that can guarantee QoS routing, while Two-hop forwards the packets to the nodes with high bandwidth which may become congested. Second, Two-hop does not take benefit of low-bandwidth nodes which may still support the QoS routing due to lower queue delay, while QOD creates full use of the resources of the nodes around a source node, and distributive ahead the packets to the APs, increasing the QoS throughput of the system.

We define the fraction of QoS throughput (QoS fraction in short) as the ratio of QoS throughput to total packet throughput. The figure shows that as the node mobility speed increases, the little bit of QoS throughput of all systems decreases. Specifically, the QoS fraction in S-Multi hop and E-AODV drops sharply, while that of QOD and Two-hop drops slightly as the average mobility of the nodes in the system increases. This is due to the reason that S-Multi hop generate longer path lengths than QOD and Two-hop, and therefore suffer from more severe link breakdown, which avert packets from arriving at APs in time. Since packet scheduling algorithm can avoid race contention as explained before, and the segment resizing algorithm can increase the scheduling feasibility of the intermediate node, its QoS fraction decreases only slightly. The packet resizing algorithm creates smaller packet size in higher node mobility, thus making more packets for a given data stream and hence more transmission overhead. We see that the QoS portion in two-hop also slightly decreases as the node mobility increases. This is because faster mobility moves to higher frequency of link breakdown and hence more dropped packets on the fly.
Performance with Various Numbers of APs:

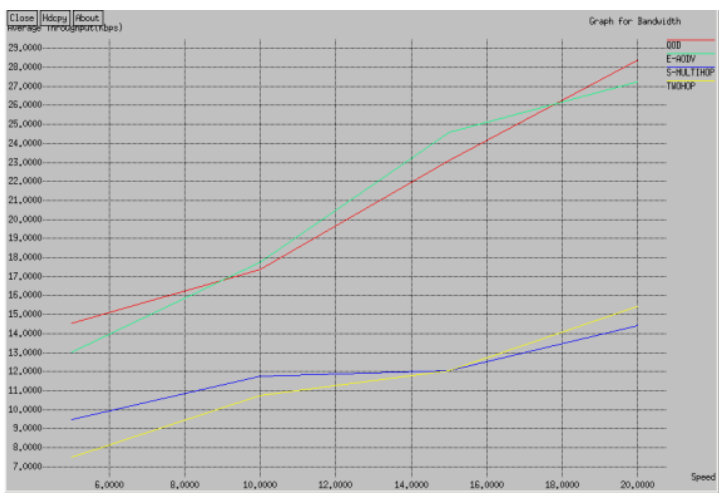

Figure5: throughput versus various access points

We define throughput versus number of access point in the different systems. The figure illustrates that the increase of APs leads to higher QoS throughput in all systems. This is for the reason that more APs help to reduce path lengths and physical distances between source nodes and AP, leading to lower packet transmission than the signal power, leading to high data transmission rate. More APs notably reduce the lengths of originally long paths to the APs in E-AODV and S-Multi hop, thus dramatically increasing their QoS throughput. In contrast, as QOD and two-hop short path, their QoS throughput increase rate is higher than those of S-Multi hop due to the same reasons explained previously, E-AODV produces high QoS throughput than S-Multi hop. When the number of the APs in the system is small, the routing path lengths of S-Multi hop and E-AODV are longer than those of QOD.

Therefore, the QoS throughput of QOD is larger than those of S-Multi hop and E-AODV and 2-hop. It is very important to see that S-Multi hop has higher QoS throughput than Two-hop when the number of APs in the system is lesser than 6 .

In this case, S-Multi hop generates much shorter path lengths. Also, S-Multi hop uses scheduling algorithm that considers buffer usage for packet routing, which decreases the packet queuing delay.

Though, Two-hop only considers channel condition for the packet routing and ignores the buffer usage, producing high-bandwidth nodes easily congested. As a result, s-multi hop makes higher QoS throughput than two hop when number of node less than 6 .

As E-AODV also suffers from congestion on the nodes close to the APs and its average path length is more than two hop as well as its throughput is also more than two hop.

As QOD can efficiently schedule the channel resources around the source node for packet forwarding, its QoS throughput remains high constantly. 
Performance with various workloads:

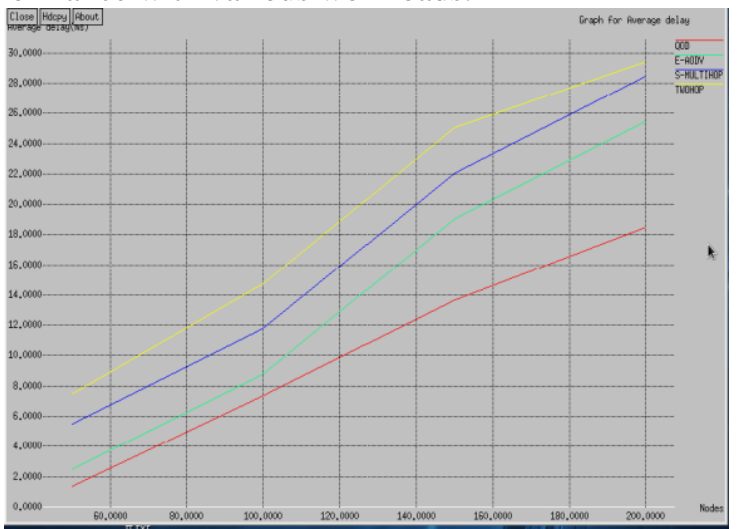

Figure6: delay versus nodes

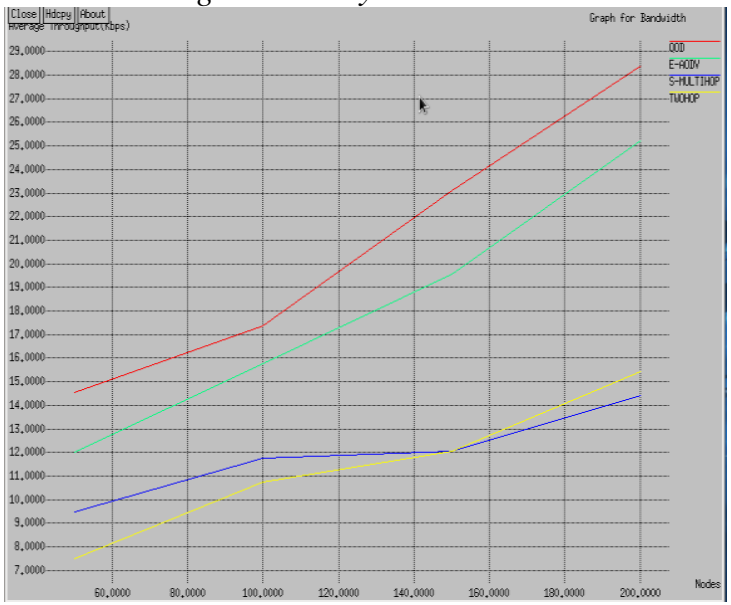

Figure7: average throughput versus various nodes with diiferent workload

Figure shows the QoS throughput of the system with different workloads in source node. Each and every node mobility speed is chosen randomly from the range 0 $\mathrm{m} / \mathrm{s}$ to the average mobility. More source nodes create more workload in the system. We monitor from both figures that as the number of source nodes increase from 0 to 180 , the QoS performance of QOD increases almost linearly. When the number of source nodes Increases to 5, the QoS throughput raises at a slower rate. In QOD, when a source node locates that all of its neighbours cannot guarantee the QoS of its packets, it stops producing new packet flows into the system based on the admission control policy. Producing more packets into the networks may further decrease the QoS performance of other source nodes. S-Multihop generates less QoS throughput increase rate than QOD, which means the system with S-Multihop drenches much earlier than that with QOD. Even though SMultihop schedules the packet forwarding by forwarding a packet to the next hop with less buffer usage, which can decrease packet buffering latency, it does not have a mechanism to avoid a node with a full buffer from receiving packets from other nodes in order to ensure the forwarding QoS. In addition, QOD's distributed packet scheduling algorithm can further reduce packet communication delay, which improves QOD's capability to handle the increasing workload in the system. As a result, QOD constantly generates higher QoS throughput than S-Multihop. The figure also shows that Two-hop has less QoS throughput increase rate than S-Multihop as the number of source nodes increases. In Two-hop, the packets are constantly forwarded to the nodes with higher communication link rate. Without any buffer management strategy, the nodes with higher communication links are very easily overloaded as the workload in the system increases. It is very exciting to see, as the number of source nodes increases, E-AODV's QoS throughput increases at first but decreases later. This is for the reason that in E-AODV, when the workload of the system increases, the possibility that two or more source nodes simultaneously reserve the same resources at a node increases due to the race condition problem. Also, the nodes closest to the APs are more likely to be congested as E-AODV does not have a resource scheduling. Therefore, the QoS throughput of E-AODV decreases in a highly loaded system.

\section{CONCLUSION}

Mobile ad-hoc networks have proven to be a better network structure for the recent generation networks. How-ever, little effort has been devoted to supporting QoS routing in MANET. In this paper, we propose a QoS-oriented distributed routing protocol (QOD) for hybrid networks to provide QoS services in a highly dynamic scenario. Taking advantage of the unique features of hybrid networks, i.e., any cast transmission and short transmission hops, QOD transforms the packet routing problem to a packet scheduling problem. In QOD, a source node directly transmits packets to an AP if the direct transmission can guarantee the QoS of the traffic. Otherwise, the source node schedules the packets to a number of qualified neighbour nodes. Specifically, QOD incorporates five algorithms. The QoS-guaranteed game theory based neighbour selection algorithm chooses qualified neighbours for packet forwarding. The distributed scheduling algorithm schedules the packet transmission to reduce the packet transmission time further. The mobility-based segment resizing algorithm resizes packets and assigns smaller packets to nodes with faster mobility to guarantee the routing QoS in a highly mobile environment.. Experimental results show that QOD can achieve high mobility-resilience and minimum delay. In the future, we plan to evaluate the performance of QOD based on the real testbed.

\section{REFERENCES}

[1] "A Majority of U.S. Mobile Users Are Now Smartphone Users,"http://adage.com/article/digital/a-majorityu-s-mobile-userssmartphone-users/241717, 2013.

[2] Sebastian Christhu Raj A, Helen Supriya M, Shanmuga Priya, "Reliable Energy Efficient Routing Algorithms In Wireless Ad Hoc Networks". International journal of scientific \& technology research, volume 3, issue 4, April 2014.

[3] Xiaojiang Du and Wei Liu "Multi-class routing and medium access control for heterogeneous mobile ad-hoc network", June 2006.

[4] . Laveen Sundararaj, Palanisamy Vellaiyan, "Delay Tolerant Networking routing as a Game Theory problem-An Overview", International Journal of Computer Networks, Volume (2), Issue(3)159, March 2010

[5] $\mathrm{H}$. Wu and X. Jia, "QoS Multicast Routing by Using Multiple Paths/Trees in Wireless Ad Hoc Networks,"Ad Hoc Networks,vol. 5, pp. 600-612, 2009. 
[6] H. Luo, R. Ramjeey, P. Sinhaz, L. Liy, and S. Lu, "UCAN: A Unified Cell and Ad-Hoc Network Architecture," Proc. ACM MobiCom, 2003

[7] P.K. Mckinley, H. Xu, A. Esfahanian, and L.M. Ni, "Unicast-Based Multicast Communication in Wormhole-Routed Direct Net-works," IEEE Trans. Parallel Data and Distributed Systems,vol. 5,no. 12, pp. $1252-1265$, Dec. 1992.

[8] H. Wu, C. Qiao, S. De, and O. Tonguz, "Integrated Cell and Ad Hoc Relaying Systems: iCAR,"IEEE J .Selected Areas in Comm.,vol. 19, no. 10, pp. 2105-2115, Oct. 2001

[9] J. Zhou and Y.R. Yang, "PAR CelS: Pervasive Ad-Hoc Relaying for Cell Systems,"Proc. IFIP Mediterranean Ad Hoc Networking Workshop (Med-Hoc-Net), 2002

[10] R. Braden, D. Clark, and S. Shenker, Integrated Services in the Internet Architecture: An Overview,IETF RFC 1633, 1994.

[11] E. Crawley, R. Nair, B. Rajagopalan, and H. Sandick, Resource Reservation Protocol RSVP, IETF RFC 2205, 1998

[12] I. Jawhar and J. Wu, "Quality of Service Routing in Mobile Ad HocNetworks,"Network Theory and Applications,Springer, 2004

[13]T. Reddy, I. Karthigeyan, B. Manoj, and C. Murthy, "Quality ofService Provisioning in Ad Hoc Wireless Networks: A Survey of Issues and Solutions,"Ad Hoc Networks,vol. 4, no. 1, pp. 83124,2006 .

[14] X. Du, "QoS Routing Based on Multi-Class Nodes for Mobile Ad Hoc Networks,"Ad Hoc Networks,vol. 2, pp. 241-254, 2004.

[15] S. Jiang, Y. Liu, Y. Jiang, and Q. Yin, "Provisioning of Adaptability to Variable Topologies for Routing Schemes in MANETs," IEEE J. Selected Areas in Comm.,vol. 22, no. 7,pp. 1347-1356, Sept. 2004.

[16] M. Conti, E. Gregori, and G. Maselli, "Reliable and Efficient Forwarding in Ad Hoc Networks," Ad Hoc Networks,vol. 4, pp. 398$415,2006$. 